Europhys. Lett., 44 (2), pp. 198-204 (1998)

\title{
Observation of an anchoring transition in a discotic liquid crystal
}

\author{
T. S. Perova ${ }^{1}$, J. K. ViJ ${ }^{1}$ and A. Kocot ${ }^{2}$ \\ ${ }^{1}$ Department of Electronic Engineering, Trinity College \\ University of Dublin - Dublin 2, Ireland \\ 2 Institute of Physics, Silesian University - Katowice, Poland
}

(received 23 July 1998; accepted 19 August 1998)

PACS. 61.30Eb - Experimental determinations of smectic, nematic, cholesteric, and other structures.

PACS. 61.30Gd - Orientational order of liquid crystals; electric and magnetic field effects on order.

PACS. 64.70Md - Transitions in liquid crystals.

\begin{abstract}
We report the anchoring transition in a discotic liquid crystal, hexapentyloxytriphenylene (HPT), from edge-on to side-on alignment in a discotic phase. The topology, easy direction of orientation and the coating of the substrate with a polymer are the determining factors for the alignment and the anchoring transition in a discotic liquid crystal.
\end{abstract}

Discotic liquid crystals are perfect examples of 2-dimensional self-organizing systems with a long-range order in the 3rd dimension. Finding reasons as to why and how such large molecules organize themselves interests physicists involved in exploring condensed matter [1]. Another reason for the continued interest in discotics lies in the potential applications of such systems. The type of orientation of the molecules inside a LC cell governs the characteristics of devices [1-4]. It has recently been shown $[1,2]$ that the columnar phase structure of discotic liquid crystals is suitable for the fast transport of photogenerated charge carriers. These results demonstrate that molecular organization in discotics plays an essential role in improving the response time of organic photoconductors; the charge mobility of hexahexylthiotriphenylene (HHTT) [1] approximates to that of an organic crystal anthracene. Consequently, it is therefore of paramount importance that causes of the surface and the surface-induced bulk alignment in discotics be explored to advance their potential for use in devices. From an extensive work carried out on nematics and smectics [5-7], certain factors (the type of the orienting layer, the mechanical rubbing or its grooving) are known to control the surface alignment. The mechanisms for the surface-induced bulk alignment are based on a) the short-range surface-molecule and then molecule-molecule interactions [8] and b) the minimization of the long-range interactions between the surface and the bulk [9].

The alignment techniques for discotic liquid crystals have so far included rotating magnetic field [10-12] and different surface treatments [13]. A strong rotating magnetic field $(\sim 2 \mathrm{~T})$ 


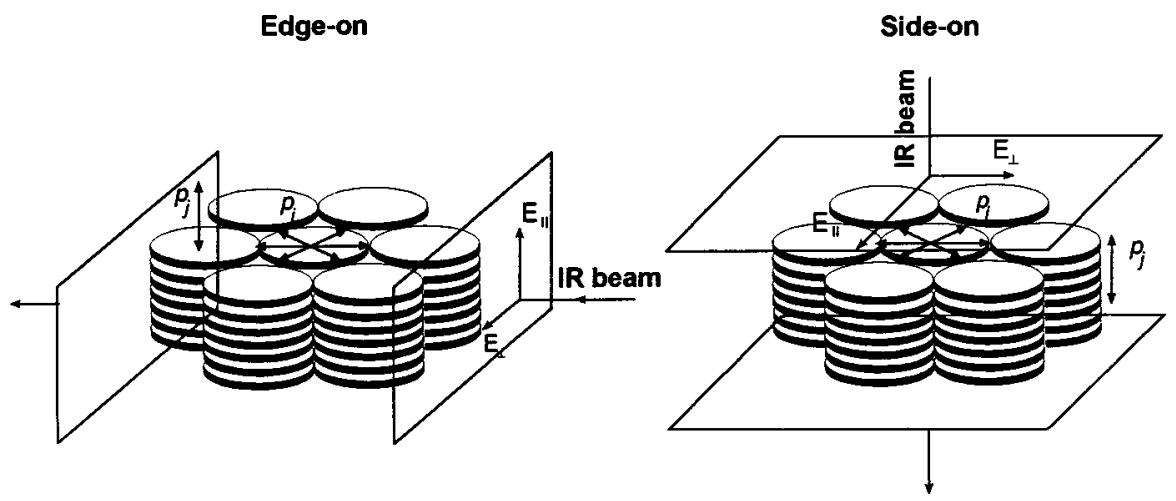

Fig. 1. - The transition dipole moments for the in-plane and out-of-plane vibrations for the side-on and edge-on alignment of HPT in the columnar phase.

is reported to produce a monodomain structure for discotics. The planar orientation of some triphenylene derivatives in columnar discotic phase on the cleavage of some crystalline materials has been observed in [13]. From these studies, however, little information is available about the influence of a surface on the alignment of discotic liquid crystals and the stability of the alignment as a function of temperature and time.

From our studies on the surface alignment and the orientations of the discotic liquid crystal as a function of the substrate, in the presence or absence of the orienting layer, we report in this letter the observation of an anchoring transition in discotics using polarized FTIR spectroscopy. We also explore the causes of planar heterogeneous and planar homogeneous alignments. The discotic liquid crystal used for these investigations is $2,3,6,7,10,11$ hexapentyloxytriphenylene (HPT) [2]. This material exhibits highly viscous mesophase at temperatures between the crystalline solid and the isotropic liquid. This is an interesting model system for discotics due to its easy alignability, capability of forming an ordered hexagonal $\left(D_{\mathrm{ho}}\right)$ mesophase [14] and being ideal for one-dimensional energy [1,2] and electron transport [4]. This discotic material is easily alignable on a substrate through simple slow cooling from the isotropic to the discotic phase. In some cases, no special surface treatment is needed for an alignment.

For investigating the bulk alignment of HPT, the IR spectra in the wave number range $600-4000 \mathrm{~cm}^{-1}$ are recorded using a Fourier transform infrared spectrometer, FTS-Biorad $60 \mathrm{~A}$ within the temperature range $300-450 \mathrm{~K}$. The IR cell containing the sample sandwiched in between the windows is thermostated within $\pm 0.1 \mathrm{~K}$.

For the analysis of an alignment two bands are selected: the aromatic C-C stretching vibrations in the range of $1500-1600 \mathrm{~cm}^{-1}$ and the $\mathrm{C}-\mathrm{H}$ out-of-plane vibrations close to $700-900 \mathrm{~cm}^{-1}$. These bands are the most sensitive indicators of ordering in discotic phase $[15$, 16] as their intensities are directly correlated with the core-core order. Transition dipole moments for the $\mathrm{C}-\mathrm{C}\left(1617 \mathrm{~cm}^{-1}\right)$ stretching vibrations are oriented parallel to the core plane, while for the $\mathrm{C}-\mathrm{H}$ out-of-plane vibration $\left(\sim 866 \mathrm{~cm}^{-1}\right)$ the transition dipole moment is oriented perpendicular to the core plane (see fig. 1). If the intensity of the C-C stretching vibration in the discotic phase is lower than in the isotropic phase, an edge-on alignment is concluded. On the contrary, if the intensity of this vibration is greater in the discotic phase than the isotropic phase, then the side-on type of alignment is established. Similarly the C-H out-of-plane vibrations can also be used to determine the alignment. 


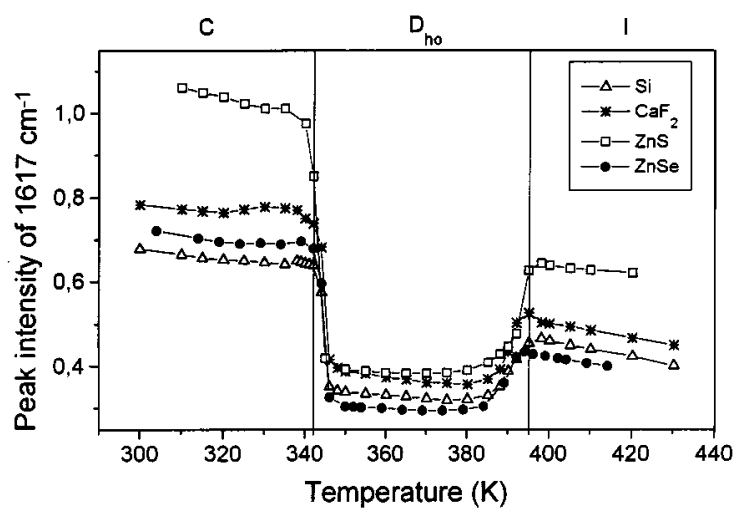

Fig. 2. - The dependence of the peak intensity of C-C aromatic stretching vibration on temperature for HPT contained in between different untreated substrates. The thickness of the cells in $\mu \mathrm{m}$ is: 12 for $\mathrm{Si}, 12$ for $\mathrm{CaF}_{2}, 17$ for $\mathrm{ZnS}$ and 10 for $\mathrm{ZnSe}$.

Orientation of the discotic LC and its stability of alignment in the discotic phase have been studied for four substrates: $\mathrm{Si}, \mathrm{CaF}_{2}, \mathrm{ZnSe}$ and $\mathrm{ZnS}$ first for clean but untreated substrates, then these substrates are coated by a polymer. Figure 2 shows an edge-on orientation of the discotic LC in the range of 340-397 K for the untreated substrates. Measurements were made with a reasonably slow temperature scan of $0.5 \mathrm{~K} / \mathrm{min}$. When the sample is kept much longer (for a few hours) in the discotic phase, the range of temperature in the discotic phase, for the edge-on orientation, is significantly reduced for $\mathrm{Si}$ and $\mathrm{CaF}_{2}$ substrates and the phase transition to side-on orientation is observed as shown in fig. 3; while the edge-on alignment for the ZnSe and ZnS remains almost unaltered. Then the orientations of discotic LC in contact with a nylon-coated substrate were probed, see fig. 4 . For the case of $\mathrm{Si}$ and $\mathrm{CaF}_{2}$ substrates only the side-on orientation is seen, while for $\mathrm{ZnSe}$ and $\mathrm{ZnS}$ the transition from the edge-on to the side-on orientation is observed with temperature (the transition for $\mathrm{ZnS}$ is found at a much lower temperature). Figure 5 shows that the stability of the edge-on orientation is improved with the increase in the thickness of the LC sample, and the transition occurs at a higher temperature for a thicker sample.

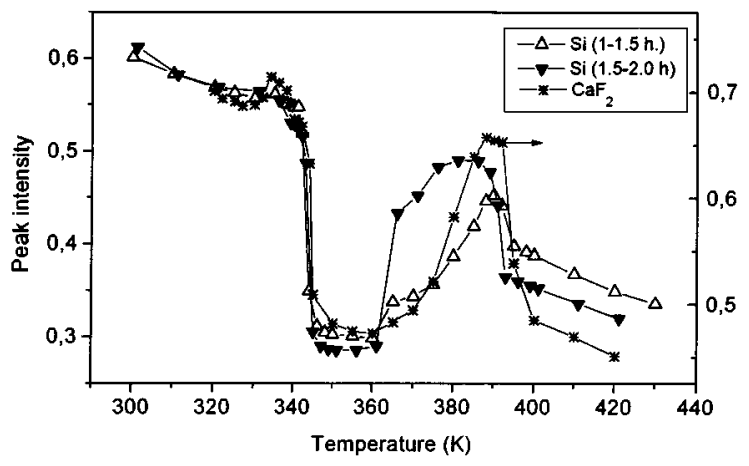

Fig. 3. - As in fig. 2, after a time period 1 to 1.5 hours $\left(\triangle \mathrm{Si} ; * \mathrm{CaF}_{2}\right)$ and $1.5-2$ hours for $\mathrm{Si}$ windows $(\nabla)$. 


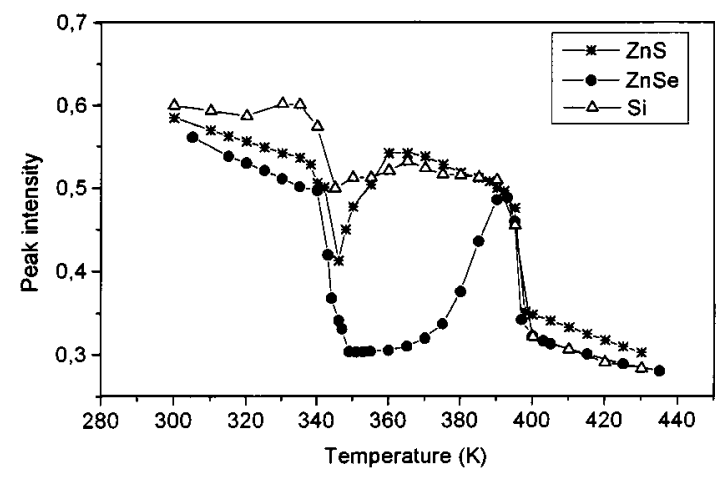

Fig. 4. - Peak intensity of the C-C aromatic vibrations vs. temperature for HPT contained in nyloncoated windows. The thickness of the cells in $\mu \mathrm{m}$ is: 10 for $\mathrm{Si}, 8$ for $\mathrm{ZnSe}$ and 9 for $\mathrm{ZnS}$.

Microscopically, the following contributions to the anchoring energy are considered. These correspond to the topology, steric, polar and dispersive (caused by van der Waals) interactions [5-7]. Except the part for topology, the other anchoring energies are primarily governed by the intermolecular interactions between the substrate and the liquid crystalline molecules. The observations show that the condition of the minimum surface energy is fulfilled if the director (axis of the column) has a planar orientation (i.e. edge-on alignment) and this type of alignment for the discotic LC is normally preferred. Whether this alignment has a homogeneous or heterogeneous character depends on whether or not a substrate has one or more than one easy direction of orientations.

$\mathrm{Si}$ is analogous to glass since a native oxide layer may also exist on the surface of $\mathrm{Si}$ windows. Though independent results of discotic alignment on Si are not available in the literature, nevertheless for glass substrates the planar heterogeneous orientation of triphenylene derivatives is observed using different techniques (X-ray, NMR etc. [10-12]). It is therefore natural to expect a similar alignment of discotic on $\mathrm{Si}$ as on a glass substrate. The characteristics of $\mathrm{CaF}_{2}$ windows are also similar to $\mathrm{Si}$ windows for the reason that three easy directions of orientation

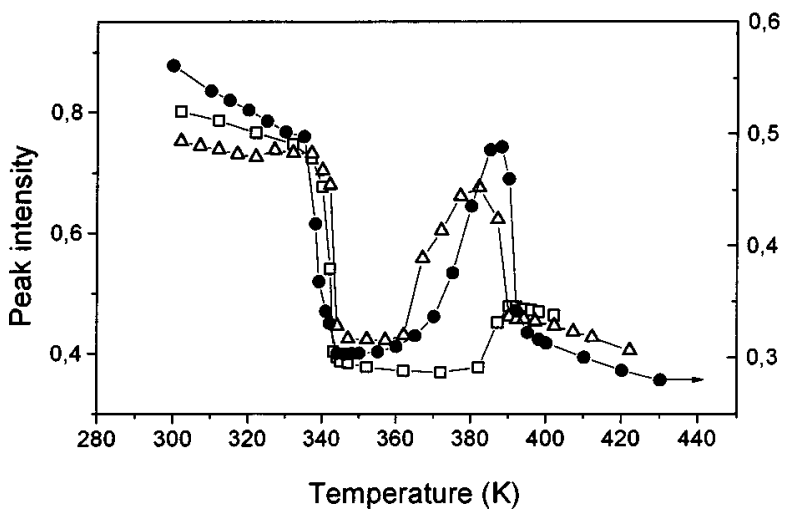

Fig. 5. - Peak intensity of the $1617 \mathrm{~cm}^{-1}$ band with $T(\mathrm{~K})$ for HPT in ZnSe windows: $\square$ without time treatment; $\triangle 1.5$ hour treatment at $T=360 \mathrm{~K}$; $\bullet$ without any waiting period for the thinner cell $(8 \mu \mathrm{m})$. 


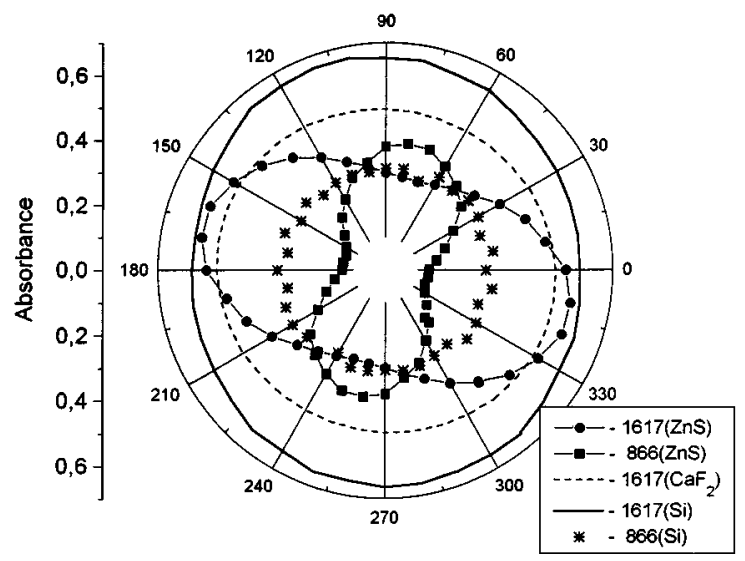

Fig. 6. - Polar plot for the intensity of the C-C in-plane vibration $\left(1617 \mathrm{~cm}^{-1}\right)$ and the C-H out-ofplane deformation $\left(866 \mathrm{~cm}^{-1}\right)$ vs. polarization angle of HPT contained in different substrates.

exist, instead of a continuous manifold of easy directions for Si [17]. For the polycrystalline ZnSe substrate, however, the situation is different. The existence of the preferable orientation (monodomain-like structure) is consistent with the determination of only one easy direction of orientation for the crystalline-cut (111) of ZnSe using X-rays [17,18]. Also ZnS shows only one easy axis of orientation [17]. The results of the polarized and oblique IR measurements are found to comply with the characteristics of the various substrates. For $\mathrm{ZnSe}$ and $\mathrm{ZnS}$, a large dichroic ratio for the vibrational bands is observed due to the existence of only one easy axis of orientation, while a low dichroic ratio for the aromatic in-plane and out-of-plane vibrations is found for the untreated $\mathrm{Si}$ and $\mathrm{CaF}_{2}$ windows (fig. 6). The orientation of the director in the plane of the windows for the last two substrates is uniformly distributed (a heterogeneous planar alignment). The weak surface anchoring energy for $\mathrm{Si}$ and $\mathrm{CaF}_{2}$ is expected and this arises from the heterogeneity of alignment on these substrates.

On considering the characteristics of the substrates, we can explain the results in terms of the anchoring phase transition. First, we remark that the transition is somewhat similar to the "local Fredericks transition" observed in nematics [19-22]. A comparison of the experimental results of discotics with nematics shows that the nature of the transition in discotics is different than in nematics. As a first step, we may adapt the theory of Dubois-Violette and de Gennes [19] for nematics to discotics. The anchoring energy per $\mathrm{cm}^{2}$ is given by

$$
F=W^{\mathrm{s}}(\theta, \varphi)+\int_{0}^{r}\left[\frac{1}{2} U(z, \theta, \varphi)+\frac{1}{2} K\left(\frac{\mathrm{d} \theta}{\mathrm{d} z}\right)^{2}\right] \mathrm{d} z .
$$

$W^{\mathrm{s}}(\theta, \varphi)$ is the surface anchoring energy due to the short-range forces. Since the edge-on alignment is observed on the untreated substrates, it is therefore safe to conclude that $W^{\mathrm{s}}(\theta, \varphi)$ due to the topology of the substrates causes this alignment. The long-range van der Waals energy, $U(z, \theta, \varphi)$, integrated over the radius $r$ also promotes the edge-on alignment, for the reason that the stability of this type of alignment is observed to increase with the sample thickness. Here $\theta$ is the angle that the director of the column makes with the normal to the substrate, $\varphi$ is the azimuthal angle with respect to an easy axis of orientation, and $z$ is measured along the normal to the substrate. The term involving the elastic constant, $K$, is the distortion energy and restores the order. For $\mathrm{Si}$ and $\mathrm{CaF}_{2}$, however, the stability of alignment is quite weak due to a degenerate anchoring of the discotic with these substrates and the fluctuations, 
with increase of temperature, drive the transition from edge-on to side-on alignment, fig. 3 . In the side-on (homeotropic) alignment the fluctuations give rise to an additional effective anchoring energy [21] (fluctuations in $\theta$ of the director in the side-on are more degenerate than in the edge-on alignment). The phase transition seems to be of the second order.

For nylon-coated substrates, the short-range interaction $W^{\mathrm{s}}(\theta, \varphi)$ is different. This arises mostly from the chemical bonds between the polymer and the discotic material. The alkane chains of the polymer seemingly lie flat on the substrate and these promote alignment of the core of discotics parallel to the surfaces. A side-on or homeotropic alignment is therefore observed, except where the topology of $\mathrm{ZnSe}$ is predominant. The van der Waals term $\int_{d}^{r} \frac{1}{2} U(z, \theta, \varphi) \mathrm{d} z$ promotes the edge-on alignment as before, but its contribution becomes smaller as the integral is now spanned over a shorter distance between $d$ and $r$, where $d$ is the thickness of the nylon film. Thus a competition between the two terms occurs, the short-range term favors the side-on, the long-range one favoring the edge-on orientation. Depending on the relative strength of the two contributions, the anchoring transition can be observed. Thus, for $\mathrm{ZnSe}$ and $\mathrm{ZnS}$ substrates a transition from the edge-on to the side-on orientation is observed, fig. 5. It is to be noted, however, that the topology of $\mathrm{ZnSe}$ (groove-like structure) increases the stability of the edge-on orientation for this substrate, so the anchoring transition is observed at a higher temperature. For $\mathrm{Si}$ and $\mathrm{CaF}_{2}$ coated with nylon, the edge-on orientation is not seen because the contribution of the short-range interactions is greater than $\int_{d}^{r} \frac{1}{2} U(z, \theta, \varphi) \mathrm{d} z$ over the entire range of the discotic phase. Figure 5 also shows that an increase of the liquid crystalline sample thickness shifts the transition towards the higher temperature. This is easily explained as the contribution of the van der Waals term increases with the increase in the sample thickness due to $r$ as the upper limit in the integral.

In summary, the anchoring transition in a discotic liquid crystal from the edge-on to the side-on alignment for a number of substrates both untreated and coated with polymer is observed. This is explained by the fluctuations that arise from the defects in the heterogeneity of alignment and a competition between the short-range surface anchoring and the long-range van der Waals interactions.

\section{$* * *$}

This work is partly funded by a research grant from Forbairt, Ireland to JKV and a Polish KBN grant 2P03B14810 to AK. We thank L. BLInOV and G. ANAN'Eva for useful discussions. Professor H. RingsdorF and Dr. O. Karthaus are thanked for synthesizing the discotic liquid crystalline material.

\section{REFERENCES}

[1] Adam D., Schuhmacher P., Simmerer J., Häussling L., Siemensmeyer K., Etzbach K. H., Ringsdorf H. and HaArer D., Nature, 371 (1994) 141.

[2] Adam D., Closs F., Frey T., Funhoff D., Haarer D., Ringsdorf H., Schuhmacher P. and Siemensmeyer K., Phys. Rev. Lett., 70 (1993) 457.

[3] Jin S., Tiefel T. H., Wolfe R., Sherwood R. C. and Mottine J. J., Science, 255 (1992) 446; Maliszewskyj N. C., Heiney P. A., Josefowicz J. Y., McCauley J. P. and Smith A. B., Science, 264 (1994) 77.

[4] Boden N., Bushby R. J. and Clements J., J. Chem. Phys., 98 (1993) 5920.

[5] Cognar J., Mol. Cryst. Liq. Cryst. (Suppl. Ser.) A, 5 (1982) 1.

[6] Jerome B., Rep. Prog. Phys., 54 (1991) 391.

[7] Blinov L. M. and Chigrinov V. G., Eletrooptic Effects in Liquid Crystal Materials (SpringerVerlag, New York) 1994.

[8] Geary J. M., Goodby J. W., Kmetz A. M. and Patel J. S., J. Appl. Phys., 62 (1987) 4100. 
[9] Berreman D. W., Phys. Rev. Lett., 28 (1972) 1683.

[10] Levelut A. M., Hardouin F., Gasparoux H., Destrade C. and Tinh N. H., J. Phys. (Paris), 42 (1981) 147.

[11] Goldfarb D., Luz Z. and Zimmermann H., J. Phys. (Paris), 42 (1981) 1303.

[12] Kranig W., Boeffel C. and Spiess H. W., Macromolecules, 23 (1990) 4061.

[13] Vauchier C., Zann A., Barny P. L., Dubois J. C. and Billard J., Mol. Cryst. Liq. Cryst., 66 (1981) 103.

[14] Destrade C., Fouchier P., Gasparoux H., Tinh N. H., Levelut A. M. and Malthete J., Mol. Cryst. Liq. Cryst., 106 (1984) 121.

[15] Perova T. S., Kocot A. and ViJ J. K., Supramolec. Sci., 4 (1997) 529.

[16] Perova T. S. and ViJ J. K., Adv. Mater., 7 (1995) 919.

[17] AnAN'EVA G. V., private communication.

[18] Anan'eva G. D., Dunaev A. A. and Merkulyaeva T. I., Russ. J. High Purity Compounds, 4 (1995) 114.

[19] Dubois-Violette E. and de Gennes P. G., J. Phys. (Paris) Lett., 36 (1975) L-255.

[20] Kats E. I., Zh. Eksp. Theor. Fiz., 70 (1976) 1394.

[21] Ryschenkov G. and Kleman M., J. Chem. Phys., 64 (1976) 404, 413.

[22] Blinov L. M. and Sonin A. A., Mol. Cryst. Liq. Cryst., 179 (1990) 13. 